\title{
Efficacy and Safety of Bevacizumab in Chinese Patients with Metastatic Colorectal Cancer
}

\author{
Li-Ming Zhu'* ${ }^{1 *}$ Ya-Zhen Zhao', Hai-Xing Ju², Lu-Ying Liu ${ }^{3}$, Lei Chen¹, Bi-Xia \\ Liu $^{1}$, Qi Xu' ${ }^{1}$, Cong Luo ${ }^{1}$, Jie-Er Ying ${ }^{1}$, Yun-Shan Yang ${ }^{1}$, Hai-Jun Zhong ${ }^{1}$
}

\begin{abstract}
Objective: To evaluate the efficacy and safety of bevacizumab in the treatment of patients with metastatic colorectal cancer (mCRC). Methods: In a single-center, observational study of 91 Chinese patients with mCRC who received bevacizumab in combination with chemotherapy was conducted. Objective response rates (ORRs), progression-free survival (PFS), overall survival (OS) and adverse events were recorded, and the relationships between various clinical factors and PFS or OS were evaluated by Cox proportional hazards models. Results: Treatment with bevacizumab and chemotherapy was effective and tolerable. Univariate analysis showed that PFS and OS were significantly associated with the Eastern Cooperative Oncology Group performance status (ECOGPS) score, duration of bevacizumab exposure, and whether chemotherapy was continued after discontinuation of bevacizumab treatment. A multivariate analysis showed that the duration of bevacizumab exposure and whether chemotherapy was continued after discontinuation of bevacizumab were independent prognostic factors for PFS and OS. Conclusion: In Chinese mCRC population, the shorter the duration of exposure to bevacizumab and chemotherapy, the worse the prognosis is.
\end{abstract}

Keywords: Metastatic colorectal cancer - chemotherapy - bevacizumab - efficacy - tolerability

Asian Pac J Cancer Prev, 15 (16), 6559-6564

\section{Introduction}

Colorectal cancer (CRC) is a malignant tumor with a high incidence all over the world, and about $1 / 4$ of patients have distant metastasis when diagnosed (Stangl et al., 1994). With the development of medical treatments and an increasing proportion of patients undergoing resection of liver metastasis, the survival of patients with metastatic CRC (mCRC) has significantly improved in recent years (Kopetz et al., 2009). For patients with unresectable mCRC, medical treatment is the most important approach. With fluorouracil monotherapy, the median survival time of patients with mCRC was reported to be $8 \sim 12$ months, but with the introduction of oxaliplatin (Oxa) and irinotecan (Iri) regimens, the median overall survival (OS) increased to 14 15 months (Colucci et al., 2005). With the subsequent use of targeted therapies such as bevacizumab, cetuximab and panitumumab, the median OS of patients with $\mathrm{mCRC}$ is further prolonged to $21.3 \sim 24.9$ months (Saltz et al., 2008; Van Cutsem et al., 2009; Douillard et al., 2010).

Bevacizumab is a humanized, anti- vascular endothelial growth factor (VEGF) monoclonal antibody. A phase III clinical trial and a large-scale observational study have shown that bevacizumab in combination with Iri or Oxa-based chemotherapy regimens and fluorouracil monotherapy are effective approaches in the treatment of some malignant tumors (Hurwitz et al., 2004; Saltz et al., 2008; Van Cutsem et al., 2009; Kozloff et al., 2009; Bendell et al., 2012). In China, bevacizumab was first approved in October, 2010 for the treatment of mCRC. In this single-center, observational study, data of Chinese patients with $\mathrm{mCRC}$ who had been treated with bevacizumab since it first became available were collected to assess its efficacy and tolerability in a routine clinical practice environment, and to investigate the relationship between various clinical factors and the survival of these patients.

\section{Materials and Methods}

\section{Study objects}

A total of 91 patients with $\mathrm{mCRC}$ who received bevacizumab in combination with chemotherapy regimens from October 1th, 2010 to January 31th, 2013 in Zhejiang Cancer Hospital were enrolled in the study, in which there were 46 males and 45 females, aged from 20 years to 74 years with the median age being 56 years. Inclusion criteria: Patients who were confirmed with $\mathrm{mCRC}$ by cytological or histological examination; aged $\geq 18$ years; with more than 1 cycle of treatment of bevacizumab in combination with chemotherapy in order to provide sufficient data for 
Table 1. Baseline Demographic and Clinical Characteristics of Patients

\begin{tabular}{|c|c|c|c|c|}
\hline Characteristics & $\begin{array}{c}\text { First-line } \\
\text { reatment }(n=42)\end{array}$ & $\begin{array}{l}\text { Second-line } \\
\text { treatment }(n=36)\end{array}$ & $\begin{array}{l}\text { Third-or fourth-l } \\
\text { treatment }(n=13)\end{array}$ & $\begin{array}{l}\text {-line All } \\
\text { ) patients }(n=91)\end{array}$ \\
\hline Gender (male/female) & $21 / 21$ & $19 / 17$ & $6 / 7$ & $46 / 45$ \\
\hline \multicolumn{5}{|l|}{ Age (years) } \\
\hline Range & $20 \sim 71$ & 39 74 & $41 \sim 71$ & $20 \sim 74$ \\
\hline$<65 / \geq 65$ & $31 / 11$ & $30 / 6$ & $11 / 2$ & $72 / 19$ \\
\hline ECOG-PS score $(0 / 1 / 2)$ & $24 / 14 / 4$ & $17 / 15 / 4$ & $2 / 8 / 3$ & $43 / 37 / 11$ \\
\hline Primary site (colon/rectum) & $26 / 16$ & $18 / 18$ & $6 / 7$ & $50 / 41$ \\
\hline History of pelvic radiotherapy (yes/no) & $3 / 39$ & $6 / 30$ & $1 / 12$ & $10 / 81$ \\
\hline Tumor differentiation (low/intermediate/ high/undifferentiated) & $9 / 20 / 3 / 10$ & $9 / 19 / 3 / 5$ & $2 / 7 / 2 / 2$ & $20 / 46 / 8 / 17$ \\
\hline Stage 4 at first visit (yes/no) & $24 / 18$ & $16 / 20$ & $4 / 9$ & $44 / 47$ \\
\hline Resection of primary lesion (yes/no) & $25 / 17$ & $29 / 7$ & $13 / 0$ & $67 / 24$ \\
\hline \multicolumn{5}{|l|}{ Chemotherapy regimens: } \\
\hline Single-agent & 22 & 5 & 9 & \\
\hline Oxa-CT & 24 & 8 & 2 & 34 \\
\hline Iri-CT & 16 & 26 & 6 & 48 \\
\hline Reason for bevacizumab discontinuation (progressive/non-progressive) & $4 / 38$ & $10 / 26$ & $7 / 6$ & $21 / 70$ \\
\hline Adjuvant chemotherapy (yes/no) & $12 / 30$ & $8 / 28$ & $3 / 10$ & $23 / 68$ \\
\hline Number of organs with metastases $(1 / \geq 2)$ & $24 / 18$ & $16 / 20$ & $3 / 10$ & $43 / 48$ \\
\hline Hypertension (yes/no) & $8 / 34$ & $11 / 25$ & $1 / 12$ & $20 / 71$ \\
\hline Ascites (yes/no) & $4 / 38$ & $3 / 33$ & $1 / 12$ & $8 / 83$ \\
\hline Palliative radiotherapy (yes/no) & $3 / 39$ & $3 / 33$ & $1 / 12$ & $7 / 84$ \\
\hline Surgery/RFA treatment (yes/no) & $5 / 37$ & $1 / 35$ & $0 / 13$ & $6 / 85$ \\
\hline Chemotherapy continued after discontinuation of bevacizumab (yes/no) & $24 / 18$ & $14 / 22$ & $3 / 10$ & $41 / 50$ \\
\hline
\end{tabular}

efficacy evaluation, including patients with progressive disease after one cycle of treatment and those who received 2-week regimens for 3 cycles or 3-week regimens for 2 cycles; expected survival time $>3$ months; Eastern Cooperative Oncology Group performance status (ECOGPS) score was 0 2; adequate reserves of bone marrow, liver and kidney functions; and provision of informed consent for participation in the study. Exclusion criteria: period from surgery to the commencement of medical treatment $<4$ weeks; other accompanying malignancies; central nervous system (CNS) metastases; uncontrolled hypertension or infection; symptomatic cardiovascular disease; coagulation disorders or receipt of anticoagulant or thrombolytic therapy; bleeding that required the use of hemostatic therapy; impaired healing of large wounds; severe peptic ulcer or intestinal obstruction; pregnancy or lactation.

The study was approved by the Ethics Committee of Zhejiang Cancer Hospital.

\section{Treatment}

The treatment of bevacizumab in combination with chemotherapy regimens given to all enrolled patients were decided by clinicians according to the disease status and discussions with the patients. Bevacizumab was given on the day before the chemotherapy regimens were administered. Depending on the chemotherapy regimen, the dosage of bevacizumab was $5 \mathrm{mg} / \mathrm{kg}$, 2 weeks as a cycle, or $7.5 \mathrm{mg} / \mathrm{kg}, 3$ weeks as a cycle. For administration, the bevacizumab was diluted with $0.9 \%$ sodium chloride injection and given by intravenous drip for $\geq 90 \mathrm{~min}$ on the first occasion. Thereafter, if well tolerated, subsequent infusions were given for $\geq 60 \mathrm{~min}$; if bevacizumabrelated toxicity such as grade II hypertension, proteinuria $\geq 2 \mathrm{~g} / 24 \mathrm{~h}$, or wound healing complications occurred, bevacizumab treatment was temporarily suspended; if grade III hypertension, nephrotic syndrome, bleeding of grade III or above, grade IV venous thrombosis, any thromboembolic events, or gastrointestinal perforation occurred, bevacizumab treatment was permanently discontinued.

According to the patient's condition, palliative radiotherapy, surgery or radiofrequency ablation (RFA) treatments were performed to relieve the symptoms.

\section{Efficacy and safety evaluations}

Efficacy evaluation included the objective response rate (ORR) of patients with measurable lesions, progression-free survival (PFS) and OS. For the short-term efficacy evaluation, the Response Evaluation Criteria in Solid Tumors (RECIST) criteria (version 1.1) was adopted (Eisenhauer et al., 2009). OS was defined as the time from the beginning of treatment to death for any reason, while PFS as the time from the beginning of treatment to death for any reason or disease progression.

The patients' medical histories were recorded and the routine examinations of blood, urine, stools and electrocardiogram (ECG) were performed within 1 week prior to treatment. All patients were given baseline imaging examinations of the chest, abdomen and pelvis, including enhanced computed tomography (CT), magnetic resonance imaging (MRI), and positron emission tomography-CT (PET-CT), within 4 weeks prior to treatment. During the treatment, imaging examinations were performed every $6 \sim 8$ weeks to evaluate the efficacy and regularly assess the bevacizumab-related toxicities. After discontinuation of bevacizumab, further assessments for bevacizumab-related toxicities were continued for 3 weeks. For the assessment and grading of bevacizumabrelated toxicities, the National Cancer Institute's Common Terminology Criteria for Adverse Events (NCI-CTCAE) criteria (version 3.0) was adopted. 
Table 2. Patients' Best Short-term Efficacy Response

\begin{tabular}{|c|c|c|c|c|c|c|}
\hline \multirow[t]{2}{*}{ Nature of treatment } & \multirow{2}{*}{$\begin{array}{c}\text { No. of } \\
\text { evaluable patients }\end{array}$} & \multicolumn{5}{|c|}{ Response [n (\%)] } \\
\hline & & CR & PR & ORR & SD & PD \\
\hline First-line $(n=42)$ & 40 & $2(5.0)$ & $22(55.0)$ & $24(60)$ & $13(32.5)$ & $3(7.5)$ \\
\hline Second-line $(n=36)$ & 31 & $0(0)$ & $11(35.5)$ & $11(35.5)$ & $16(51.6)$ & $4(12.9)$ \\
\hline Third- or fourth-line $(n=13)$ & 11 & $0(0)$ & $1(9.1)$ & $1(9.1)$ & $7(63.6)$ & $3(27.3)$ \\
\hline Total $(\mathrm{n}=91)$ & 82 & $2(2.4)$ & $34(41.5)$ & $36(43.9)$ & $36(43.9)$ & $10(12.2)$ \\
\hline
\end{tabular}

Table 3. Analysis of the Impact of Various Clinical Factors on PFS and OS

\begin{tabular}{|c|c|c|c|c|c|}
\hline \multirow[t]{2}{*}{ Factor } & \multirow[t]{2}{*}{$\mathrm{n}$} & \multicolumn{2}{|c|}{ PFS } & \multicolumn{2}{|c|}{ OS } \\
\hline & & $\mathrm{HR}(95 \% \mathrm{Cl})$ & $P$-value & $\mathrm{HR}(95 \% \mathrm{Cl})$ & $P$-value \\
\hline Female gender & 45 & $0.92(0.58 \sim 1.46)$ & 0.730 & $1.34(0.73 \sim 2.46)$ & 0.344 \\
\hline Age $\geq 65$ years & 19 & $0.69(0.39 \sim 1.24)$ & 0.212 & $0.74(0.33 \sim 1.68)$ & 0.475 \\
\hline ECOG-PS score 1 or 2 & 48 & $2.31(1.44 \sim 3.70)$ & $<0.001$ & $2.90(1.50 \sim 5.60)$ & 0.002 \\
\hline Primary site in colon & 50 & $0.86(0.54 \sim 1.36)$ & 0.517 & $1.61(0.85 \sim 3.05)$ & 0.141 \\
\hline Not at stage IV at first visit & 47 & $1.24(0.79 \sim 1.96)$ & 0.355 & $0.88(0.65 \sim 1.19)$ & 0.407 \\
\hline \multicolumn{6}{|l|}{ Chemotherapy regimens: } \\
\hline Iri-CT & 48 & $1.19(0.72 \sim 1.95)$ & 0.501 & $1.00(0.53 \sim 1.92)$ & 0.989 \\
\hline Single-agent & 9 & $1.56(0.71 \sim 3.45)$ & 0.268 & $0.86(0.29 \sim 2.56)$ & 0.788 \\
\hline Bevacizumab application time $>3.4$ months & 45 & $0.38(0.24 \sim 0.61)$ & $<0.001$ & $0.41(0.21 \sim 0.79)$ & 0.007 \\
\hline $\begin{array}{l}\text { Chemotherapy not continued after } \\
\text { discontinuation of bevacizumab }\end{array}$ & 50 & $2.36(1.48 \sim 3.80)$ & $<0.001$ & $2.60(1.34 \sim 5.06)$ & 0.005 \\
\hline \multicolumn{6}{|l|}{ Nature of treatment: } \\
\hline Second-line & 36 & $1.83(1.09 \sim 3.10)$ & 0.023 & $1.65(0.86 \sim 3.19)$ & 0.134 \\
\hline Third- or fourth-line & 13 & $2.69(1.38 \sim 5.24)$ & 0.004 & $1.28(0.50 \sim 3.26)$ & 0.605 \\
\hline No hypertension present & 71 & $1.42(0.79 \sim 2.53)$ & 0.243 & $1.50(0.69 \sim 3.28)$ & 0.306 \\
\hline No use of combined local treatment & 78 & $1.42(0.73 \sim 2.78)$ & 0.306 & $2.20(0.78 \sim 6.17)$ & 0.135 \\
\hline Primary lesion unresected & 24 & $1.08(0.64 \sim 1.80)$ & 0.779 & $2.20(1.14 \sim 4.23)$ & 0.018 \\
\hline Number of organs with metastases $\geq 2$ & 48 & $1.85(1.16 \sim 2.95)$ & 0.010 & $1.84(0.99 \sim 3.42)$ & 0.056 \\
\hline No ascites present & 83 & $0.65(0.31 \sim 1.36)$ & 0.255 & $0.35(0.16 \sim 0.80)$ & 0.013 \\
\hline No adjuvant chemotherapy administered & 68 & $1.08(0.64 \sim 1.83)$ & 0.764 & $1.83(0.86 \sim 3.86)$ & 0.115 \\
\hline
\end{tabular}

\section{Statistical analysis}

Data analysis was performed using SPSS software package (version 17.0). Survival time was estimated by the Kaplan-Meier method, and comparisons of PFS and OS among subgroups were performed with log-rank test. Univariate analysis was conducted using Cox proportional hazards model, and based on the results of this analysis, variables with a statistical significance level of $P<0.2$ were chosen for the multivariate model in which the influence of various factors on PFS and OS was analyzed using a backward stepwise regression multivariate Cox proportional hazards model. Differences were considered statistically significant when $P<0.05$.

\section{Results}

\section{Patient characteristics}

Nitety-one patients met the inclusion/exclusion criteria and were eligible for this observational study. Oxaliplatinbased chemotherapy (Oxa-CT) regimens (CAPOX, mFOLFOX6 or FOLFOX4) were given to 34 patients, while irinotecan-based chemotherapy (Iri-CT) regimen (FOLFIRI) to 48, and single-agent therapy (fluorouracil, capecitabine or raltitrexed) to 9 .

Until the final follow-up date (July 31 th, 2013), the median follow-up duration was 17.4 months (range: 6 33.4 months). Eleven patients continued treatment after this date, but 80 had discontinued bevacizumab treatment for a variety of reasons, including treatment refusal or personal reasons in 45 , disease progression in 21 , liver metastasis surgery or RFA in 6, bevacizumab-related toxicity in 4, and appendicitis, intestinal obstruction, or liver abscess complications in 4 . The median duration of bevacizumab exposure for all 91 patients was 3.4 months (range: $0.5 \sim 16.5$ months). Following discontinuation of bevacizumab, 41 patients continued to receive chemotherapy, while 50 discontinued their chemotherapy at the same time as discontinuation of bevacizumab. The baseline demographic and clinical characteristics of patients are shown in Table 1.

\section{Efficacy}

A short-term efficacy evaluation was able to be performed in 82 patients with measurable lesions. Complete response (CR), partial response (PR), objective response rate (ORR), stable disease (SD) and progressive disease (PD) were analyzed (Table 2).

Until the final follow-up date, 75 patients had progressive disease and 42 had died. The median PFS of all patients was 6.4 months (95\% CI, 5.0 7.8 months) and the median OS was 20.2 months (95\% CI, 16.3 24.1 months). In patients who received first- or second-line treatments, the median PFS was 8.6 months and 5.5 months, and the median OS was 21.9 months and 17.1 months, respectively. For patients who received third- or fourth-line treatments, the median PFS was 3.6 months, but the median OS had not been reached.

\section{Bevacizumab-related adverse effects}

Bevacizumab-related adverse events in 91 patients 
Table 4. Multivariate Analysis of Clinical Factors

\begin{tabular}{lccccr}
\hline Factor & \multicolumn{2}{c}{ PFS } & & \multicolumn{2}{c}{ OS } \\
\cline { 2 - 3 } \cline { 5 - 6 } & HR $(95 \% \mathrm{Cl})$ & P-value & & HR $(95 \% \mathrm{Cl})$ & $P$-value \\
\hline Bevacizumab application time $>3.4$ months & $0.303(0.178 \sim 0.516)$ & 0.000 & $0.344(0.175 \sim 0.675)$ & 0.002 \\
Chemotherapy not continued after discontinuation of bevacizumab & $1.685(1.015 \sim 2.797)$ & 0.044 & $3.538(1.741 \sim 7.189)$ & 0.000 \\
Nature of treatment: & & & & - \\
Second-line & $1.603(0.923 \sim 2.782)$ & 0.094 & - & - \\
Third- or fourth-line & $2.141(1.086 \sim 4.221)$ & 0.028 & - & - \\
Number of organs with metastases $\geq 2$ & $2.114(1.218 \sim 3.670)$ & 0.008 & - & - \\
Primary lesion unresected & - & - & $4.413(2.087-9.328)$ & 0.000 \\
\hline
\end{tabular}

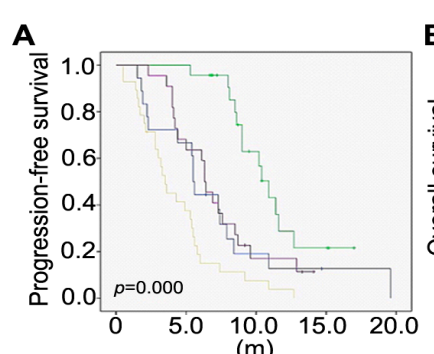

(m)

B

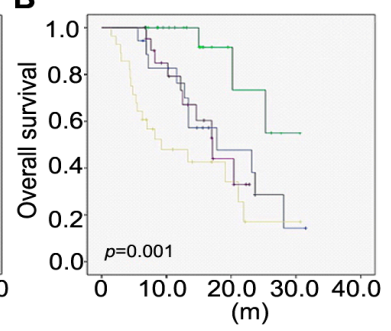

Chemotherapy continuation group after discontinuation of Bev when Bev exposure was $\leq 3.4$ months Che Chemotherapy discontinuation group after discontinuation of Bev when Bev exposure was $\leq 3.4$ months Chemotherapy discontinuation group after discontinuation of Bev when Bev exposure was >3.4 month + Censored + Censored Censored + Censored

Figure 1. Impact of Duration of Bevacizumab Administration and Suspension of Chemotherapy after Discontinuation of Bevacizumab on PFS and OS

are recorded. Four patients stopped the treatment due to bevacizumab-related toxicities, including 1 case of grade III hypertension, 1 case of grade I and 1 of grade IV gastrointestinal perforation, and 1 case of grade II hematochezia. Seven patients temporarily suspended bevacizumab treatment due to toxicities, including 4 with wound healing complications, 1 with grade III proteinuria, 1 with left renal vein thrombosis, and 1 with right brachial vein thrombosis. In the remaining 80 patients, there were 10 cases of grade 1-2 Hypertension, 5 cases of grade I IIproteinuria and 21 cases of grade I IIbleeding. No cases of congestive heart failure or reversible posterior leukoencephalopathy syndrome were observed.

\section{Analysis of prognostic factors}

The univariate analysis was performed on 15 variables including gender, age, ECOG-PS score, primary tumor site, whether the patient was at phase IV at the first visit, the chemotherapy regimens administered, the bevacizumab application time, etc. The results of this analysis showed that PFS was closely associated with the nature of the treatment and the number of organs with metastases, and OS was closely associated with whether the primary lesion was resected and whether ascites existed. Additionally, both PFS and OS were significantly related to the ECOGPS score, the bevacizumab application time, and whether chemotherapy was continued after discontinuation of bevacizumab (Table 3).

All variables with a statistical significance level of $P<0.2$ in the univariate analysis were then further analyzed using a backward stepwise regression multivariate Cox proportional hazards model. The results of this analysis showed that the bevacizumab application time and whether chemotherapy was continued after discontinuation of bevacizumab were major independent prognostic factors influencing PFS and OS (Table 4). Kaplan-Meier analysis indicated that with a shorter duration of bevacizumab administration and suspension of chemotherapy after discontinuation of bevacizumab, the patients' PFS and OS were worse (Figure 1).

\section{Discussion}

In the AVF2107g study of patients with mCRC conducted in 2004, bevacizumab in combination with irinotecan/fluorouracil/leucovorin (IFL) significantly improved the patients' ORR, PFS and OS in comparison with the IFL regimen alone (Hurwitz et al., 2004). In the subsequent NO16966 study of bevacizumab in combination with Oxa-CT (XELOX or FOLFOX4) for first-line treatment of mCRC patients (Saltz et al., 2008), the MAX study of bevacizumab in combination with capecitabine monotherapy or capecitabine and mitomycin for first-line treatment of mCRC patients, and the AVEX study of bevacizumab in combination with single-agent capecitabine therapy for first-line treatment of elderly mCRC patients (Cunningham et al., 2013), positive primary endpoint results were obtained. Similarly, the phase III, randomized ARTIST study of first-line treatment of Chinese patients with mCRC confirmed that bevacizumab in combination with a modified IFL regimen significantly improved efficacy in comparison with the modified IFL regimen alone (Guan et al., 2011).

In a study of second-line treatment of mCRC, the E3200 clinical trial showed that bevacizumab in combination with FOLFOX4 comprehensively improved the ORR, PFS and OS of patients with mCRC in comparison with the FOLFOX4 regimen alone (Giantonio et al., 2007). The ML18147 study of bevacizumab cross-line treatment demonstrated that after first-line bevacizumabbased treatment had been changed to a second-line chemotherapy regimen, the continued use of bevacizumab still significantly improved the primary endpoint (OS) (Bennouna et al., 2013) of the study. In studies of thirdor fourth-line bevacizumab-based treatment, some phase II clinical studies and small-sample retrospective studies have reported that third-line bevacizumab-based treatment of mCRC can also achieve a good level of efficacy, with a median OS in the range 9.1 14.1 months (Chen et al., 2006; Kang et al., 2009; Park et al., 2012).

In the phase III clinical studies of bevacizumab-based 
regimens versus chemotherapy alone reviewed above, adverse events experienced with bevacizumab included hypertension, bleeding, proteinuria, gastrointestinal perforation, and arterial and venous thrombosis, but the overall occurrence rate of these toxicities was low and most patients were able to tolerate them. In the present study, the data on Chinese patients with mCRC treated at our center indicated that bevacizumab is effective in various lines of treatments, with a low adverse event occurrence rate and a low permanent discontinuation rate due to adverse events (3.3\%).

In clinical trials of drugs for cancer treatment, the usual reasons for discontinuation of treatment are disease progression or intolerable toxicity, but in routine clinical practice, we also need to consider the patient's economic status and the intention of treatment. Thus, there are additional factors that may affect the prognosis in routine clinical practice.

In the phase III NO16966 clinical trial, the median duration of bevacizumab exposure was 190 days, and the median PFS times of the intention-to-treat populations of the bevacizumab and placebo treatment groups were 9.4 months and 8.0 months, respectively (HR=0.83; $P=0.0023)$. When a group who continued bevacizumab treatment compared with the placebo group, the median PFS times were 10.4 months and 7.9 months, respectively $(\mathrm{HR}=0.63 ; P<0.0001)$, indicating that the longer the duration of bevacizumab exposure, the more obvious the difference of median PFS times between the 2 groups. In the phase III CAIRO3 study, in which patients initially received 6 cycles of induction therapy with a capecitabine/oxaliplatin regimen (CAPOX) combined with bevacizumab, maintenance treatment with capecitabine in combination with bevacizumab was compared with simple observation. The PFS time was 4.1 months in the observation group and 8.5 months in the maintenance treatment group, which was significantly different $(P<0.001)$ (Koopman et al., 2013). In the phase III, non-inferiority SAKK 41/06 study in which patients received 4 6 months of bevacizumab in combination with chemotherapy as first-line treatment, the time-toprogression (TTP) of a bevacizumab maintenance therapy group was compared with an observation group. In the bevacizumab maintenance therapy group, the TTP was 4.1 months, while that in the observation group was 2.9 months, HR=0.74 (95\% CI, 0.57 0.95; $P=0.47)$, which failed to reach the preset HR value of 0.727 (Koeberle et al., 2013). Overall, these prospective phase III studies indirectly suggest that sustained bevacizumab exposure can produce a greater survival benefit.

In other observational studies, the BRiTE study, which was based on a routine clinical practice environment, found that the median duration of first-line bevacizumabbased treatment was 5.4 months as compared with a median duration of chemotherapy of 5.7 months (Kozloff et al., 2009), while the First BEAT study reported that the average duration of bevacizumab exposure was 260 days (Van Cutsem et al., 2009). However, neither of these studies explored relationships between the duration of bevacizumab exposure and the patients' prognoses.

Based on chemotherapy toxicity considerations in the treatment of mCRC, a series of phase III clinical studies have been designed. The OPTIMOX1 study which compared the standard FOLFOX4 regimen administered until disease progression (arm A) with an investigational regimen consisting of FOLFOX7 (a simplified fluorouracil and leucovorin regimen with high-dose oxaliplatin) for 6 cycles followed by fluorouracil monotherapy and then 24 weeks later or after progression, resumption of the FOLFOX7 regimen ( $\operatorname{arm} \mathrm{B}$ ), found that while the efficacy of the 2 regimens (PFS and survival times) was similar, grade III or IV toxicity was observed less commonly with the FOLFOX7 regimen (48.7\% of patients vs. $54.4 \%$ with the FOLFOX4 regimen) and fewer patients experienced grade III sensory neuropathy (13.3\% vs. $17.9 \%$ with the FOLFOX4 regimen). The OPTIMOX2 study showed that the duration of disease control (DDC) and median PFS of patients in a chemotherapy cessation group were significantly lower than those in a chemotherapy maintenance group, but the median OS of patients in the chemotherapy cessation group was worse than that of patients in the chemotherapy maintenance group (Chibaudel et al., 2009). These results were confirmed by the COIN trial (Adams et al., 2011). This study showed that although the toxicity experienced by a planned chemotherapy cessation (intermittent chemotherapy) group was significantly reduced compared with a chemotherapy maintenance group, the efficacy of intermittent chemotherapy could not be guaranteed, and it could not be concluded that the OS of the intermittent chemotherapy group was non-inferior to that of the chemotherapy maintenance group (Adams et al., 2011). Therefore, to reduce toxicity, complete cessation of chemotherapy for mCRC patients prior to disease progression is not advised.

In China, most patients who receive bevacizumab do so at their own expense, and the high cost of the drug restricts its clinical use. In addition, because many patients have concerns over toxicity with chemotherapy, the durations of bevacizumab and chemotherapy exposure may not be adequate. For these patients, whether a reduction in the duration of bevacizumab or chemotherapy exposure may have an important clinical impact remains to be clarified in further studies.

In the present study, the univariate analysis was performed on 15 variables and the results showed that the ECOG-PS score, the bevacizumab application time, whether chemotherapy was continued after discontinuation of bevacizumab, the nature of treatment, and the number of organs with metastases were associated with PFS, while OS was associated with the ECOG-PS score, the bevacizumab application time, whether chemotherapy was continued after discontinuation of bevacizumab, whether the primary lesion was resected, and whether ascites existed. The major factors influencing PFS and OS were determined using a backward stepwise regression multivariate Cox proportional hazards model. The results of this multivariate analysis showed that the nature of treatment and the number of organs with metastases were independent prognostic factors of PFS, while the primary lesion resected or not was an independent prognostic factor of OS. The bevacizumab application time and whether 
chemotherapy was continued after discontinuation of bevacizumab were independent prognostic factors for both PFS and OS.

In conclusion, our data have shown that bevacizumab in combination with chemotherapy was effective and tolerable in the treatment of Chinese patients with mCRC and in a routine clinical practice environment, the shorter the duration of bevacizumab and chemotherapy exposure, the worse the prognosis was. Among all independent prognostic factors affecting PFS or OS, only the duration of bevacizumab and chemotherapy exposure can be artificially adjusted, which is important for patients' survival. However, the sample size of this study is small and as it is a retrospective analysis, some selection bias may have occurred. Therefore, these conclusions require further confirmation in prospective clinical trials.

\section{Acknowledgements}

The research is supported by Zhejiang Province Public Technology Research and Social Development Project Grants 2013C33206.

\section{References}

Adams RA, Meade AM, Seymour MT, et al (2011). Intermittent versus continuous oxaliplatin and fluoropyrimidine combination chemotherapy for first-line treatment of advanced colorectal cancer: results of the randomised phase 3 MRC COIN trial. Lancet Oncol, 12, 642-53.

Bendell JC, Bekaii-Saab TS, Cohn AL, et al (2012). Treatment patterns and clinical outcomes in patients with metastatic colorectal cancer initially treated with FOLFOXbevacizumab or FOLFIRI-bevacizumab: results from ARIES, a bevacizumab observational cohort study. Oncologist, 17, 1486-95.

Bennouna J, Sastre J, Arnold D, et al (2013). Continuation of bevacizumab after first progression in metastatic colorectal cancer (ML18147): a randomised phase 3 trial. Lancet Oncol, 14, 29-37.

Colucci G, Gebbia V, Paoletti G (2005). Phase III randomized trial of FOLFIRI versus FOLFOX4 in the treatment of advanced colorectal cancer: a multicenter study of the Gruppo Oncologico Dell'Italia Meridionale. J Clin Oncol, 23, 4866-75.

Chen HX, Mooney M, Boron M, et al (2006). Phase II multicenter trial of bevacizumab plus fluorouracil and leucovorin in patients with advanced refractory colorectal cancer: an NCI Treatment Referral Center Trial TRC-0301. J Clin Oncol, 24, 3354-60.

Chibaudel B, Maindrault-Goebel F, Lledo G, et al (2009). Can chemotherapy be discontinued in unresectable metastatic colorectal cancer? The GERCOR OPTIMOX2 Study. J Clin Oncol, 27, 5727-33.

Cunningham D, Lang I, Marcuello E, et al (2013). Bevacizumab plus capecitabine versus capecitabine alone in elderly patients with previously untreated metastatic colorectal cancer (AVEX): an open-label, randomised phase 3 trial. Lancet Oncol, 14, 1077-85.

Dirican A, Varol U, Kucukzeybek Y, et al (2014). Treatment of metastatic colorectal cancer with or without bevacizumab: can the neutrophil/lymphocyte ratio predict the efficiency of bevacizumab? Asian Pac J Cancer Prev, 15, 4781-6.

Douillard JY, Siena S, Cassidy J, et al (2010). Randomized, phase III trial of panitumumab with infusional fluorouracil, leucovorin, and oxaliplatin (FOLFOX4) versus FOLFOX4 alone as first-line treatment in patients with previously untreated metastatic colorectal cancer: the PRIME study. $J$ Clin Oncol, 28, 4697-705.

Eisenhauer EA, Therasse P, Bogaerts J, et al (2009). New response evaluation criteria in solid tumours: revised RECIST guideline (version 1.1). Eur J Cancer, 45, 228-47.

Giantonio BJ, Catalano PJ, Meropol NJ, et al (2007). Bevacizumab in combination with oxaliplatin, fluorouracil, and leucovorin (FOLFOX4) for previously treated metastatic colorectal cancer: results from the Eastern Cooperative Oncology Group Study E3200. J Clin Oncol, 25, 1539-44.

Guan ZZ, Xu JM, Luo RC, et al (2011). Efficacy and safety of bevacizumab plus chemotherapy in Chinese patients with metastatic colorectal cancer: a randomized phase III ARTIST trial. Chin J Cancer, 30, 682-9.

Hurwitz H, Fehrenbacher L, Novotny W, et al (2004). Bevacizumab plus irinotecan, fluorouracil, and leucovorin for metastatic colorectal cancer. N Engl J Med, 350, 2335-42.

Kopetz S, Chang GJ, Overman MJ, et al (2009). Improved survival in metastatic colorectal cancer is associated with adoption of hepatic resection and improved chemotherapy. J Clin Oncol, 27, 3677-83.

Kozloff M, Yood MU, Berlin J, et al (2009). Clinical outcomes associated with bevacizumab-containing treatment of metastatic colorectal cancer: the BRiTE observational cohort study. Oncologist, 14, 862-70.

Kang BW, Kim TW, Lee JL, et al (2009). Bevacizumab plus FOLFIRI or FOLFOX as third-line or later treatment in patients with metastatic colorectal cancer after failure of 5-fluorouracil, irinotecan, and oxaliplatin: a retrospective analysis. Med Onvcol, 26, 32-7.

Koopman M, Simkens LH, Ten Tije AJ, et al (2013). Maintenance treatment with capecitabine and bevacizumab versus observation after induction treatment with chemotherapy and bevacizumab in metastatic colorectal cancer (mCRC): the phase III CAIRO3 study of the Dutch Colorectal Cancer Group (DCCG). J Clin Oncol, 31, Abstract 3502.

Koeberle D, Betticher DC, Von Moos R, et al (2013). Bevacizumab continuation versus no continuation after firstline chemo-bevacizumab therapy in patients with metastatic colorectal cancer: a randomized phase III noninferiority trial (SAKK 41/06). J Clin Oncol, 31, Abstract 3503.

Park LC, Lee HS, Shin SH, et al (2012). Bevacizumab as a second- or later-line of treatment for metastatic colorectal cancer. World J Gastroenterol, 18, 1104-9.

Saltz LB, Clarke S, Díaz-Rubio E, et al (2008). Bevacizumab in combination with oxaliplatin-based chemotherapy as firstline therapy in metastatic colorectal cancer: a randomized phase III study. J Clin Oncol, 26, 2013-9.

Stangl R, Altendorf-Hofmann A, Charnley RM, et al (1994). Factors influencing the natural history of colorectal liver metastases. Lancet, 343, 1405-10.

Van Cutsem E, Köhne CH, Hitre E, et al (2009). Cetuximab and chemotherapy as initial treatment for metastatic colorectal cancer. N Engl J Med, 360, 1408-17.

Van Cutsem E, Rivera F, Berry S, et al (2009). Safety and efficacy of first-line bevacizumab with FOLFOX, XELOX, FOLFIRI and fluoropyrimidines in metastatic colorectal cancer: the BEAT study. Ann Oncol, 20, 1842-7.

Wu Q, Shi Y, Chen L, et al (2013). Effect and safety of bevacizumab-containing chemotherapy treatment in Chinese patients with metastatic colorectal cancer. Onco Targets Ther, 6, 485-90. 